\title{
When Pandemics Collide: the Interplay of Obesity and COVID-19
}

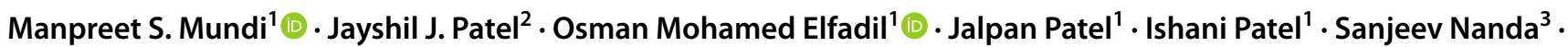 \\ Ryan T. Hurt ${ }^{1,3,4}$
}

Accepted: 24 September 2021 / Published online: 4 November 2021

(C) The Author(s), under exclusive licence to Springer Science+Business Media, LLC, part of Springer Nature 2021

\begin{abstract}
Purpose of Review The COVID-19 pandemic has been associated with significant morbidity and mortality worldwide. In addition to those with advanced age and co-morbidities such as heart disease or cancer, obese individuals have also had very high rates of hospitalization, critical illness, need for ventilator support, as well as mortality. A number of factors associated with obesity have led to devastating consequences as these two pandemics have interacted.

Recent Findings Obese individuals through a combination of structural and cellular level changes have greater risk of ischemic heart disease, diabetes, cancer, and respiratory disease, which are themselves risk-factors for acquiring COVID-19 disease. These structural changes also result in increased intra-abdominal and intra-thoracic pressure as well as a restrictive lung physiology that leads to reduction in total lung capacity, functional residual capacity, and increase in airway hyperreactivity. Adipose tissue is also impacted in obese individuals leading to local as well as systemic inflammation, which can contribute to increased release of free fatty acids and systemic insulin resistance. Additionally, angiotensin-converting enzyme 2 and dipeptidyl peptidase 4, which act as receptors for SARS-CoV-2 are also significantly increased in obese individuals. Summary The present manuscript reviews these structural, immune, and molecular changes associated with obesity that make obese individuals more vulnerable to acquiring severe COVID-19 and more challenging to manage associated complications.
\end{abstract}

Keywords COVID-19 $\cdot$ SARS-CoV-2 $\cdot$ Obesity $\cdot$ Inflammation

\section{Introduction}

At the end of 2019, a novel severe acute respiratory syndrome coronavirus 2 (SARS-CoV-2) virus causing respiratory disease (COVID-19) was detected in Wuhan, China $[1 \bullet \bullet]$. In the following months, cases were detected worldwide, leading the World Health Organization (WHO) to

This article is part of the Topical Collection on Nutrition and Obesity

Manpreet S. Mundi

mundi.manpreet@mayo.edu

1 Division of Endocrinology, Diabetes, Metabolism and Nutrition, Mayo Clinic, 200 First St SW, Rochester, MN 55905, USA

2 Division of Pulmonary \& Critical Care Medicine, Medical College of Wisconsin, WI, Milwaukee, USA

3 Division of General Internal Medicine, Mayo Clinic, Rochester, MN, USA

4 Division of Gastroenterology and Hepatology, Mayo Clinic, Rochester, MN, USA declare a pandemic. The medical community gained better understanding of risk factors for severe disease, which often led to hospitalization, use of critical care services, and death. Early reports from the Chinese Center for Disease Control and Prevention reported a 2.3\% case fatality. In the initial reports, older age, a history of cardiovascular disease, diabetes, chronic respiratory disease, hypertension, and cancer were deemed risk factors for severe disease $[1 \bullet \bullet]$. Body mass index (BMI) and its correlation with disease severity or mortality was not initially described, but this soon changed when data emerged from other countries.

Among 20,133 patients hospitalized with SARS-CoV-2 infection across 208 hospitals in the United Kingdom, obesity was identified in $10.5 \%$ [2]. Worse, obesity was a strong predictor of mortality (HR 1.33; 95\% confidence interval (CI): 1.19 to 1.49) after adjusting for other comorbidities. Between March 1 and April 8, 2020, 5279 patients at NYU Langone Health tested positive for SARS-CoV-2 [3]. Of these, 2741 (51.9\%) required hospitalization, 990 (36.1\%) developed critical illness requiring intensive care unit (ICU) services, and 665 (24.3\%) died. In multi-variate analysis, 
obesity (especially a BMI $>40 \mathrm{~kg} / \mathrm{m}^{2}$ ) emerged as a risk factor for both hospital admission (OR 2.5; 95\% CI:1.8 to 3.4) and critical illness requiring ICU services (OR 1.5; 1.0 to 2.2). In another report from New York City, among 3615 individuals who tested positive for SARS-CoV-2, 775 (21\%) had a BMI of $30-34 \mathrm{~kg} / \mathrm{m}^{2}$ and $595(16 \%)$ had a BMI of $35 \mathrm{~kg} / \mathrm{m}^{2}$ or higher [4]. Among patients under 60 years old, those with a BMI of $30-34 \mathrm{~kg} / \mathrm{m}^{2}$ were $2.0(1.6-2.6)$ times as likely to be admitted to the hospital and $1.8(1.2-2.7)$ times as likely to be admitted to the ICU, as compared to those with normal range BMI. In a cohort from Mexico of 51,633 SARS-CoV-2 positive cases and 5332 related deaths (10.3\%), the obese, as compared to non-obese, had a higher rate of mortality (13.5\% versus $9.4 \%$ ), critical illness (5.0\% versus $3.3 \%$ ), and ventilator support (5.2\% versus $3.3 \%$ ) [5]. Data from France found a higher rate of obesity in those SARS-CoV-2 patients who were critically ill and required mechanical ventilation (Odds ratio of 7.36 [1.63-33.14] comparing $\mathrm{BMI} \geq 35$ vs. $<25$ ) $[6 \bullet]$.

These data highlight the devastating impact of one pandemic (obesity) on another (COVID-19). Obese individuals may have a compounded risk for acquiring more severe COVID-19 disease. First, individuals who are obese undergo gross structural and cellular level changes which puts them at greater risk for ischemic heart disease, diabetes, cancer, and respiratory disease, which are themselves risk-factors for acquiring COVID-19 disease. Second, obesity-specific structural changes can make caring for obese patients who acquire COVID-19 disease logistically challenging. Finally, there may be a link between obesity and SARS-CoV-2 specific receptors found in adipose tissue, possibly rendering obese individuals more susceptible to acquiring more severe disease. This manuscript reviews the structural, inflammatory, and molecular changes induced by obesity and aims to propose mechanisms by which obese patients are more vulnerable to acquiring severe COVID-19 disease and its associated complications and challenges to care.

\section{Obesity Related Structural Changes}

As an individual gains weight, structural changes to the body occur. In terms of fat deposition, there are two main patterns: central (apple-shaped) with deposition in the thorax, abdomen, and visceral tissues -or- peripheral (pear-shaped) with deposition of fat in the hips, thighs, limbs/extremities, and subcutaneous tissue (Fig. 1). As fat begins to deposit in the abdominal cavity, intra-abdominal pressure increases. Sugerman et al. evaluated intra-abdominal pressure in 84 subjects with BMI $\geq 35 \mathrm{~kg} / \mathrm{m}^{2}$ undergoing bariatric surgery and compared it to 5 non-obese controls. Obese individuals had significantly higher intra-abdominal pressure, as compared to non-obese controls $\left(18 \pm 0.7\right.$ versus $7 \pm 1.6 \mathrm{~cm} \mathrm{H}_{2} \mathrm{O}$, $\mathrm{p}<0.001)$ [7]. In addition, increased abdominal pressure correlated with abdominal diameter and obesity related comorbidities with the highest intra-abdominal pressure being found in those with 3 or more obesity related co-morbidities.

Central fat deposition alters the mechanical properties of the chest wall and generates and/or accentuates (pre-existing) lung disorders $[8,9,10 \bullet]$. Lung disorders can be classified as restrictive or obstructive. The pathophysiology and differences between restrictive and obstructive lung diseases is beyond the scope of this review. As an oversimplification, restrictive disorders prevent the lungs from fully expanding
Fig. 1 Central versus Peripheral adiposity. By permission of Mayo Foundation for Medical Education and Research. All rights reserved
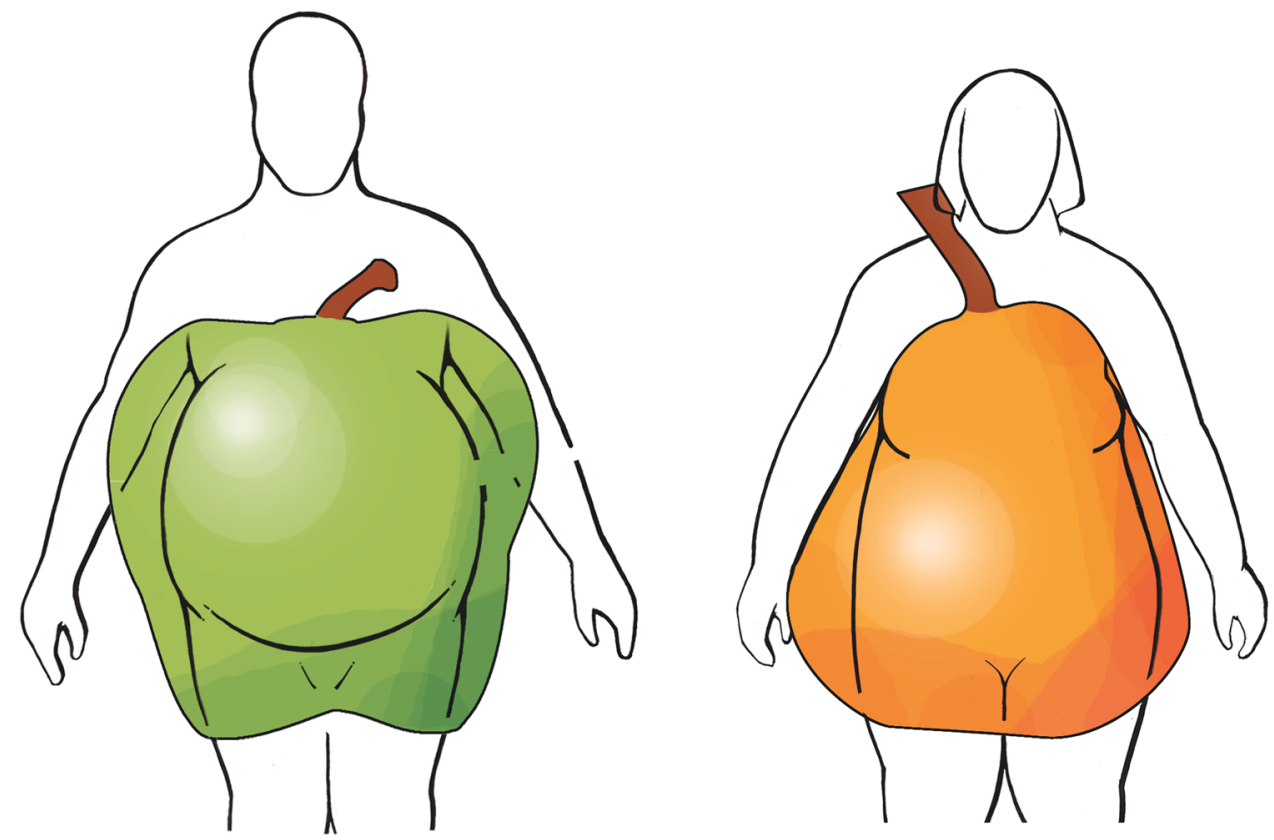
because of disease in the chest wall, weak muscles (e.g., diaphragm), and/or impaired nervous system function. In contrast, obstructive disorders are characterized by narrowing of airways, which prevents incomplete exhalation. Pulmonary function testing, which includes spirometry, lung volumes, and diffusion capacity testing are adjuncts to history and examination to differentiate between obstructive and restrictive lung disease.

Obesity is associated with restrictive physiology. Fat deposition increases the load respiratory muscles must overcome to raise the chest wall and prevents it from expanding. Fat deposition in the abdominal cavity increases intraabdominal pressure and restricts downward movement of the diaphragm [10•]. Diaphragmatic motion is impaired, further impairing chest wall expansion. As a result, lung volume tests reveal reduced total lung capacity (TLC) and functional residual capacity (FRC) (Fig. 2). In a retrospective study, Jones et al. found a significant decline in mean predicted FRC with corresponding increase in BMI (with BMI 20-25 kg/m²: $103.1 \pm 15.5$, BMI 25-30: 89.2 \pm 14.1 , BMI 30-35: 78.3 \pm 13.1 , BMI 35-40 72.2 \pm 12.9 , and BMI $>40$ : $66.6 \pm 12.3)$ [11]. These changes translated to a reduction in FRC of 3\% for each unit increase in BMI from 20 to $30 \mathrm{~kg} /$ $\mathrm{m}^{2}$ and $1 \%$ decrease for each unit increase of BMI above $30 \mathrm{~kg} / \mathrm{m}^{2}$.

Since airway diameter is dependent on lung volume and obese individuals breathe at lower lung volumes, early airway closure ensues, which may exacerbate pre-existing obstructive lung diseases such as asthma or chronic obstructive pulmonary disease (COPD). Behazin et al. compared the esophageal and airway pressure in 50 obese $\left(\geq 35 \mathrm{~kg} / \mathrm{m}^{2}\right)$ and 10 non-obese subjects who underwent surgical intervention [12]. They found airway pressure was significantly higher in obese individuals and was above $1.3 \mathrm{~cm} \mathrm{H}_{2} \mathrm{O}$ in most cases, indicating most of the airways were closed [12]. In obese subjects, both esophageal and gastric pressures were also elevated compared to non-obese controls. These changes were associated with impaired respiratory system resistance and compliance in the obese individuals, compared to non-obese $(0.032 \pm 0.008$ versus $0.053 \pm 0.007, p<0.001)$. To evaluate the relationship of obesity with airway hyper-responsiveness (AHR), participants from the Normative Aging Study who underwent multiple methacholine challenge tests were evaluated. Those who tested positive in subsequent methacholine challenge test after initial negative study were matched to individuals who tested negative for both tests [13]. After controlling for factors such as age, smoking status, and pack years of smoking, the study found obesity was associated with significantly higher odds ratio (7.5; 95\% CI 1.5 to 37.8 ) of subsequent positive methacholine challenge. More importantly, those who had the highest quintile of BMI increase $(0.4$ to $1.9 \mathrm{~kg} /$ $\mathrm{m}^{2}$ higher per year) had the highest odds of developing AHR compared to those individuals with minimal weight gain. A meta-analysis that included seven epidemiologic studies with over 300,000 subjects noted a dose response effect of increased BMI and asthma [14]. Compared to normal weight individuals, the odds-ratio of asthma was significantly increased in both overweight $(1.38 ; 95 \%$ CI 1.17-1.62) and obese (1.92; 95\% CI 1.43-2.59) individuals. These changes contribute to many respiratory

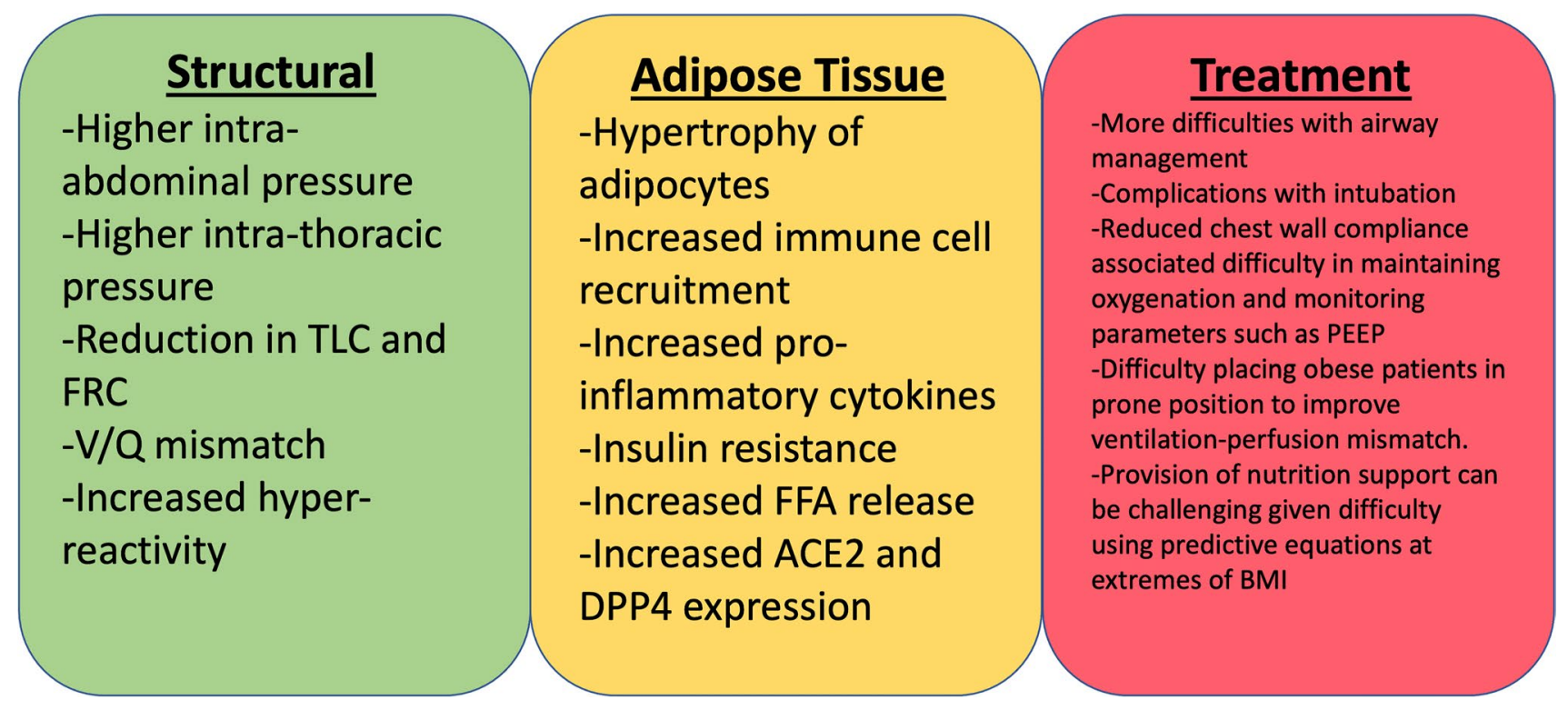

Fig. 2 Summary of obesity related changes that predispose to poor outcomes with COVID-19. By permission of Mayo Foundation for Medical Education and Research. All rights reserved 
symptoms observed in obesity, including shortness of breath, orthopnea, wheezing, and alterations in breathing patterns $[7,10 \bullet, 15]$.

\section{Obesity Related Inflammation}

\section{Adipocyte Inflammation}

Obesity also significantly alters adipose tissue with one of the main changes affecting the immune cells and inflammation Fig 2. With excessive caloric intake, adipose tissue can expand through hypertrophy (increased adipocyte size), hyperplasia (increased adipocyte number), or both [16]. In animal models, it seems that after initial adipocyte hypertrophy reaching the upper volume limit of adipocytes, de novo adipogenesis is initiated allowing for further increase in energy storage [17]. However in adult humans, studies have noted that adipocyte number and turnover remains relatively stable, indicating that adipocyte hypertrophy may be the main mechanism of increase in energy storage [16, 18].

This growth through hypertrophy can unfortunately have significant metabolic and inflammatory consequences. Hypertrophic adipocytes show necrotic-like abnormalities impeding function, inducing inflammation, and even increasing cell death [19]. Hypertrophic adipocytes are associated with increased expression and secretion of pro-inflammatory cytokines, leading to recruitment of various immune cells, including macrophages [20]. Additionally, hypertrophic adipose tissue tends to be hypoxic due to relative deficiency of vasculature, leading to further activation of inflammation and fibrosis [21].

This increase in inflammatory cells in adipose tissue such as the M1 macrophage leads to further increase in production of cytokines such as interleukin-6 (IL-6) and tumor necrosis factor - alpha (TNF- $\alpha$ ), contributing to both local and systemic inflammation associated with obesity [22]. In adipose tissue, these pro-inflammatory cytokines induce insulin resistance and stimulate lipolysis, which tends to be already increased in hypertrophic adipocytes, leading to increased release of free fatty acids (FFAs) [23]. As an example, high levels of TNF- $\alpha$ interfere with insulin signaling through inhibition of phosphorylation of insulin receptor substrate 1 and 2 [24]. Adipocyte insulin resistance is further worsened by anatomical changes such as disrupted cortical actin structures and impaired insulin-dependent GLUT-4 trafficking to the plasma membrane, contributing to further release of FFA [16]. In this state of overnutrition, the excess FFA are taken up by tissues such as the liver and muscle, leading to lipid accumulation, lipotoxicity, and insulin resistance in those tissues [23].

\section{Systemic Inflammation}

Unfortunately, the pro-inflammatory state associated with obesity is not just localized to adipose tissue and results in systemic inflammation as well [25]. The inflammatory cytokines released by adipose tissue begin to activate inflammatory cells such as M1 macrophages and pro-inflammatory T helper type 1 cells systemically [26]. Additionally, excess intake of nutrients, especially saturated free fatty acids can also lead to stimulation of toll-like receptors (TLR) expression in dendritic cells and macrophages [26]. TLR are pattern-recognition receptors that detect the presence of pathogenic microbial infection and play a crucial role in the first line of host-defense. Typically, TLR bind to lipopolysaccharides of cell membranes, but can also be activated in response to circulating saturated fatty acids such as palmitate $(\mathrm{C} 16: 0)$ and stearate (C18:0). This activation can then lead to central leptin and insulin resistance as well as systemic inflammation. Recent study demonstrated that central administration of saturated fatty acids resulted in activation of pro-inflammatory gene expression in a TLR-dependent manner in the hypothalamus [27].

\section{Immune Response}

One of the hallmark manifestation of COVID-19 has been a "cytokine storm" or uncontrolled over-production of soluble inflammatory markers leading to acute respiratory distress syndrome (ARDS) [28•]. Early reports began to demonstrate higher levels of cytokines such as IL-1B, IL-6, IL-12, TNF- $\alpha$ and chemokines (CXCL10, CCL2) in patients admitted to ICU with COVID-19 compared to those who were not [28]. It was also noted that a key feature of COVID-19 especially in critically ill patients was lymphopenia, which often preceded pulmonary symptoms [29]. T lymphocytes including both CD4 and CD8 subtypes were especially reduced and tended to recover over months [30]. In addition to a decrease in peripheral lymphocytes, atrophy in secondary lymphoid organs (lymph nodes and spleen) is also noted with immunohistochemical staining showing decreased numbers of CD4 and CD8 positive and natural killer (NK) T-cells [30]. In lieu of CD4 and CD8 lymphocytes, the majority of inflammatory cells infiltrating the lungs are monocytes and macrophages [31].

Under normal innate immune system, macrophages, monocytes, dendritic cells, and neutrophils express pattern recognition receptors (PRR) on their membranes, which detect pathogen-associated molecular patterns (PAMPs) [30]. This detection triggers a signaling process that leads to expression of pro-inflammatory cytokine-inducing transcription factors as well as activation of interferon regulatory factors that help mediate an antiviral response. Another set of pathogen recognition sensors are present in the cytosol 
and detect endogenous danger-associated molecular patterns (DAMPs). Binding of DAMPs activates the formation of inflammasomes, which are multi-protein cytoplasmic complexes that leads to activation of pro-inflammatory cytokines [32]. Under normal conditions, virus-infected cells are destroyed by NK cells and CD8 positive T-cells leading to apoptosis of the antigen-presenting cells. However, in situations where there is a defect in the cytolytic activity, a prolonged and exaggerated interaction between innate and adaptive immune cells occurs, leads to pro-inflammatory cytokines being released in an unrestrained manner, resulting in a cytokine storm and ultimately ARDS, multi-organ failure, and death.

Coronaviruses such as SARS-CoV-2 have developed a number of strategies that allow them to evade the immune system resulting in a prolonged infection and/or the maladaptive immune response seen in COVID-19. One mechanism is the production of vesicles that contain double membranes without PRR, allowing replication without antiviral immune response and viral clearance [33]. Accessory viral proteins also bind to double-stranded RNA leading to a down regulation of antiviral cytokines and a net upregulation of pro-inflammatory cytokines. Rapid viral replication may also induce pyroptosis, which is a form of programmed cell death associated with rapid plasma-membrane rupture and release of pro-inflammatory intracellular contents [34].

This maladaptive immune response may be worsened in obese individuals. As discussed above, nutrition excess leads to an activation of macrophages and pro-inflammatory state with excess circulating pro-inflammatory cytokines. Additionally, animal models have demonstrated a more intense reaction to pro-inflammatory cytokines resulting in cytokine storm and death. Ramos-Muniz et al. inoculated lean and obese mice with Francisella tularensis, the causative agent associated with tularemia and known to result in overproduction of inflammatory cytokines [35]. They noted that inoculation with a dose resulting in $25 \%$ and $50 \%$ death in lean mice resulted in $65 \%$ and $100 \%$ death in obese mice. Although, bacterial load was not different among the lean and obese mice, there was significantly higher plasma levels of cytokines such as IL- 6 and TNF- $\alpha$. In fact, gene expression of these pro-inflammatory cytokines was upregulated 15- to 65 -fold in obese mice compared to lean mice.

In addition to more intense inflammatory reaction, deficiency in activation and function of $\mathrm{CD} 4$ and $\mathrm{CD} 8$ positive T-lymphocytes has also been demonstrated in obese individuals in the setting of viral infections such as H1N1 influenza A virus [36, 37]. Interrogation of T-cells noted reduced number and those remaining cells showing increased differentiation associated with impairment in antiviral functionality and response to antigen presentation [36]. These changes in immune response also result in delayed clearance and higher peak viral loads. Maier et al. evaluated influenza-A virus shedding over three seasons in Managua, Nicaragua [38]. They noted that in symptomatic obese individuals, viral shedding was $42 \%$ longer than their symptomatic lean counterparts. In asymptomatic individuals, viral shedding in obese adults was $104 \%$ longer than in lean adults. Despite the increase viral load, the decreased activation of $\mathrm{T}$ cells also results in reduced long-term antibody titer and subsequent immunity in obese individuals. Sheridan et al. reported that compared to their normal weight counterparts, obese individuals had lower antibody titer and decreased CD8 T cell activation 12 months after receiving influenza vaccine [39]. Another study demonstrated that despite undergoing vaccination, obese individuals had double the risk $(9.8 \%$ versus $5.1 \%$ ) of developing influenza [40].

\section{Obesity and SARS-CoV-2 Receptors}

Coronaviruses such as SARS-CoV-2 are positive stranded RNA viruses that have a unique structure with surfaceanchored spike protein that facilitates entry into cells. The spike protein is present as a trimer, with three $\mathrm{S} 1$ heads sitting on top of S2 membrane fusion stalk [41]. S1 contains a receptor binding domain (RBD) that specifically recognizes the angiotensin-converting enzyme 2 (ACE2) facilitating entry. ACE2 is present in arterial and venous endothelial cells in all organs including nasopharynx, oral mucosa, nasal mucosa, stomach, colon, liver, kidney, and the brain [42]. Additionally, significant expression is noted on lung alveolar endothelial cells and enterocytes of the small intestine [42]. In addition to ACE2, the RBD also has affinity for multiple human proteins including dipeptidyl peptidase 4 (DPP4), which is expressed on the apical surfaces of epithelium of various organs such as the lung and liver [43]. In fact, SARSCoV-2 may be unique in its ability to bind to both ACE2 and DPP4 compared to previous deadly coronaviruses.

Another unique feature has been binding affinity to these human proteins with studies revealing conflicting results based on methods used [41]. This paradox may occur because the RBD can be either in a standing-up state that enables it to bind to its receptor or in a lying-down state that does not bind the host receptors. Compared to SARS-CoV spike, which tends to be mostly in the standing position, the SARS-CoV-2 spike protein tends to be mostly in the lying position. Since the RBD is the most immunogenic region of the whole spike, this allows SARS-CoV-2 to evade host immune system and lead to prolonged recovery time [41]. SARS-CoV-2 counters the RBD in a lying position by having its spike protein proteolytically activated at its $\mathrm{S} 1 / \mathrm{S} 2$ cleavage site by proteases such as human transmembrane protease serine 2 (TMPRSS2), furin, elastase, factor X, and trypsin [44]. 
This mechanism of cell binding and subsequent cell entry by SARS-CoV-2 has tremendous implications for obese individuals. Adipose tissue expresses ACE2 with higher mRNA expression in visceral compared to subcutaneous adipose tissue. More importantly, in animal models with overfeeding and weight gain, within 1 week, ACE2 mRNA expression, activity, and protein levels were increased in adipose tissue [45]. This increase in mRNA expression continued after 4 months of over-feeding. In humans a recent evaluation of 5457 Icelanders from the Age, Gene/Environment Susceptibility Reykjavic Study noted that ACE2 levels were significantly increased in overweight and obese individuals compared to their lean counterparts [46]. Other studies evaluating ACE2 levels from human bronchial epithelial cells have also noted increased levels in overweight or obese individuals compared to their normal weight counterparts $[47,48]$.

Similar to ACE2 levels, DPP4 levels are significantly increased in obesity and adipose tissue is a significant source of circulating DPP4. In a study of 196 subjects of varying $\mathrm{BMI}$ and insulin resistance, it was noted that DPP4 expression positively correlated with BMI with visceral adipose tissue consistently having higher expression compared to subcutaneous expression [49]. Net DPP4 release was higher in obese individuals compared to lean, with insulin-sensitive obese individuals having significantly lower circulating levels compared to obesity matched insulin resistant subjects. In addition to these receptors, adipose tissue also expresses proteases TMPRSS2 and furin, facilitating both entry as well as exit of virus particles by priming the spike protein [50]. By having these viral receptors and proteases, it is speculated that adipose tissue can serve as a viral reservoir thus propagating viral infection along with the strong immune response that leads to tissue damage and multi-organ failure [51].

\section{Challenges in Management of the Critically III Obese Patient}

Physical challenges related to the obese critically ill patients with COVID-19 disease are similar to that observed prepandemic Fig 2. Greater adipose tissue in obese patients can lead to difficulties with airway management, proper positioning (e.g., for bag-mask ventilation), and landmark identification (e.g., for peripheral or central venous catheter placement). Frat et al. compared complications of endotracheal intubation in 82 morbidly obese critically ill patients (mean BMI $42 \pm 6 \mathrm{~kg} / \mathrm{m}^{2}$ ) to 124 non-obese patients (mean BMI $24 \pm 4 \mathrm{~kg} / \mathrm{m}^{2}$ ). Morbidly obese patients, as compared to non-obese, had more peri-intubation difficulties (15 versus $6 \%, \mathrm{p}<0.05)$ and post-extubation stridor $(15$ versus $3 \%$, $\mathrm{p}<0.05)$ [52]. Since FRC is reduced in obese patients, obese patients may develop atelectasis and resultant hypoxemia. Reduced chest wall compliance poses a unique challenge in monitoring lung compliance and titration of ventilatory parameters like positive-end expiratory pressure (PEEP). In addition, ultrasound strategies to gauge circulating volume status may not be feasible. Early mobility is a key component of good ICU care. Mobilizing the obese patient is labor intensive and may be associated with complications, including greater fall risk.

COVID-19 specific challenges with the obese patients include issues with prone positioning. SARS-CoV-2 is known to cause lung injury, often manifesting as acute respiratory distress syndrome. Prone positioning is a method to enhance ventilation-perfusion matching in patients with ARDS and was adopted early in the pandemic [53, 54]. In fact, numerous studies reported the benefits of awake prone positioning in patients with COVID-19 disease [55-57]. Spinal fracture is the only absolute contraindication for prone positioning [54]. Obesity, including morbid obesity, is not a contraindication. The PROSEVA trial, which randomized 466 patients with severe ARDS to undergo prone-positioning of at least $16 \mathrm{~h}$ or maintain in the supine position, found improved 28- and 90-day mortality in those who underwent prone positioning [53]. The median BMI in the PROSEVA trial was $28 \mathrm{~kg} / \mathrm{m}^{2}$ (interquartile range 21-36). However, patients in the PROSEVA trial were mechanically ventilated and sedated. Data on difficulties in moving an awake obese patient with COVID-19 disease from the supine to prone positioning are not available, but we speculate that it may be labor intensive requiring coordinated efforts between a multidisciplinary ICU team. Turning a patient may lead to transient hemodynamic instability and hypoxemia. In an awake obese patient, transient hypoxemia may not be welltolerated due to reduced FRC.

Pharmacologic management and nutrition support are non-physical challenges in the critically ill obese. The pharmacology of weight-based medications used commonly in the ICU setting are not well studied in obese patients. Due to increased extracellular water and fat in obesity, medication dosing poses a unique challenge. Finally, determining precise caloric requirements is challenging in obese patients and weight-based equations may lead to over- or underfeeding. Predictive equations, such as Harris-Benedict, are not accurate for estimating resting energy expenditure (REE) and were not validated in severe obesity. Even though indirect calorimetry is the most accurate method for measuring REE in obese patients, the COVID-19 pandemic has limited its use out of concern for protecting healthcare workers, limiting personal protective equipment use, and limiting contamination. In mechanically ventilated obese patients with COVID-19 disease, the optimal timing, dose, composition, and up-titration rate is not defined. ICU practitioners often utilize guidance borne out of the critically ill non-COVID-19 
population. The awake prone obese patient poses a unique challenge. Anecdotal reports suggest many have limited or no volitional intake. It is unclear if these patients benefit from early nasogastric tube placement and initiation of enteral nutrition or parenteral nutrition [58].

\section{Conclusions}

The interplay of obesity and COVID-19 has had devastating consequences with increased morbidity and mortality. With the implementation of widespread vaccination, the world is eagerly awaiting the resolution of COVID-19 pandemic. However, the obesity pandemic continues to increase unabated especially in the United States, where the prevalence is expected to rise to $50 \%$ in adults by 2030 . As obesity is associated with changes throughout the body including structural changes as well as those associated with adipose tissue itself that make the obese individual more susceptible to developing obesity related co-morbidities and the next viral pandemic. Additionally, treatment of obese individuals can be more challenging, contributing to worse outcomes. With resolution of COVID-19 pandemic, our focus must remain on combating the obesity pandemic with similar unified effort and urgency in order to prevent further morbidity and mortality.

\section{Declarations}

Conflict of Interest The authors declare no competing interests.

Human and Animal Rights and Informed Consent This article does not contain any studies with human or animal subjects performed by any of the authors.

Financial Disclosure Manpreet S. Mundi has research grant from Fresenius Kabi, Nestle, Realfood Blends, and VectivBio and is a consultant for Baxter. Ryan T. Hurt, M.D. PhD is a consultant for Nestle and has research grant from Zealand. Jayshil Patel, M.D. is a consultant for Baxter. Osman Mohamed Elfadil, Jalpan Patel, Ishani Patel, and Sanjeev Nanda have no disclosures to report.

\section{References}

Papers of particular interest, published recently, have been highlighted as:

- Of importance

$\bullet$ Of major importance

1.•• Wu Z, McGoogan JM. Characteristics of and Important Lessons From the Coronavirus Disease 2019 (COVID-19) Outbreak in China: Summary of a Report of 72314 Cases From the Chinese Center for Disease Control and Prevention. JAMA. Published online February 24, 2020. https://doi.org/10.1001/jama.2020.2648.
Initial report characterizing the demographics and outcomes of patients with COVID-19 from China.

2. Docherty AB, Harrison EM, Green CA, et al. Features of 20133 UK patients in hospital with covid-19 using the ISARIC WHO clinical characterisation protocol: prospective observational cohort study. BMJ. 2020;369. https://doi.org/10. 1136/bmj.m1985.

3. Petrilli CM, Jones SA, Yang J, et al. Factors associated with hospital admission and critical illness among 5279 people with coronavirus disease 2019 in New York City: prospective cohort study. BMJ. 2020;369. https://doi.org/10.1136/bmj. m1966.

4. Lighter J, Phillips M, Hochman S, et al. Obesity in patients younger than 60 years is a risk factor for Covid-19 hospital admission. Clin Infect Dis. https://doi.org/10.1093/cid/ciaa415.

5. Bello-Chavolla OY, Bahena-López JP, Antonio-Villa NE, et al. Predicting mortality due to SARS-CoV-2: a mechanistic score relating obesity and diabetes to COVID-19 outcomes in Mexico. J Clin Endocrinol Metab. Published online May 31, 2020. https://doi.org/10.1210/clinem/dgaa346.

6. Simonnet A, Chetboun M, Poissy J, et al. High prevalence of obesity in severe acute respiratory syndrome coronavirus-2 (SARS-CoV-2) requiring invasive mechanical ventilation. Obesity (Silver Spring). Published online April 9, 2020. https://doi. org/10.1002/oby.22831. A retrospective cohort study demonstrating correlation between BMI and mechanical ventilation in patients who are positive for SARS-CoV-2.

7. Sugerman H, Windsor A, Bessos M, Wolfe L. Intra-abdominal pressure, sagittal abdominal diameter and obesity comorbidity. J Intern Med. 1997;241(1):71-9. https://doi.org/10.1046/j.13652796.1997.89104000.x.

8. Mundi MS, Karpyak MV, Koutsari C, Votruba SB, O’Brien PC, Jensen MD. Body fat distribution, adipocyte size, and metabolic characteristics of nondiabetic adults. J Clin Endocrinol Metab. 2010;95(1):67-73. https://doi.org/10.1210/jc.2009-1353.

9. Shah M, Hurt RT, Mundi MS. Phenotypes of obesity: how it impacts management. Curr Gastroenterol Rep. 2017;19(11):55. https://doi.org/10.1007/s11894-017-0598-1.

10. Peters U, Dixon AE. The effect of obesity on lung function. Expert Rev Respir Med. 2018;12(9):755-67. https://doi.org/10. 1080/17476348.2018.1506331 Excellent review of the impact of obesity on lung function including the mechanics of the lung and chest wall, as well as changes that lead to asthma and airway hyper-responsiveness.

11. Jones RL, Nzekwu M-MU. The effects of body mass index on lung volumes. Chest. 2006;130(3):827-33. https://doi.org/10. 1378/chest.130.3.827.

12. Behazin N, Jones SB, Cohen RI, Loring SH. Respiratory restriction and elevated pleural and esophageal pressures in morbid obesity. J Appl Physiol (1985). 2010;108(1):212-8. https://doi. org/10.1152/japplphysiol.91356.2008.

13. Litonjua A, Sparrow D, Celedon J, DeMolles D, Weiss S. Association of body mass index with the development of methacholine airway hyperresponsiveness in men: the normative aging study. Thorax. 2002;57(7):581-5. https://doi.org/10.1136/thorax. 57.7.581.

14. Beuther DA, Sutherland ER. Overweight, obesity, and incident asthma. Am J Respir Crit Care Med. 2007;175(7):661-6. https:// doi.org/10.1164/rccm.200611-17170C.

15. Sin DD, Jones RL, Man SFP. Obesity is a risk factor for dyspnea but not for airflow obstruction. Arch Intern Med. 2002;162(13):1477. https://doi.org/10.1001/archinte.162.13. 1477.

16. Choe SS, Huh JY, Hwang IJ, Kim JI, Kim JB. Adipose tissue remodeling: its role in energy metabolism and metabolic 
disorders. Front Endocrinol (Lausanne). 2016;7:10.3389/ fendo.2016.00030.

17. Wang QA, Tao C, Gupta RK, Scherer PE. Tracking adipogenesis during white adipose tissue development, expansion and regeneration. Nat Med. 2013;19(10):1338-44. https://doi.org/10.1038/ nm.3324.

18. Spalding KL, Arner E, Westermark PO, et al. Dynamics of fat cell turnover in humans. Nature. 2008;453(7196):783-7. https:// doi.org/10.1038/nature06902.

19. Giordano A, Murano I, Mondini E, et al. Obese adipocytes show ultrastructural features of stressed cells and die of pyroptosis. J Lipid Res. 2013;54(9):2423-36. https://doi.org/10.1194/jlr. M038638.

20. Jernås M, Palming J, Sjöholm K, et al. Separation of human adipocytes by size: hypertrophic fat cells display distinct gene expression. FASEB J. 2006;20(9):1540-2. https://doi.org/10. 1096/fj.05-5678fje.

21. Trayhurn P. Hypoxia and adipose tissue function and dysfunction in obesity. Physiol Rev. 2013;93(1):1-21. https://doi.org/10. 1152/physrev.00017.2012.

22. Gregor MF, Hotamisligil GS. Inflammatory mechanisms in obesity. Annu Rev Immunol. 2011;29(1):415-45. https://doi.org/10. 1146/annurev-immunol-031210-101322.

23. Rutkowski JM, Stern JH, Scherer PE. The cell biology of fat expansion. J Cell Biol. 2015;208(5):501-12. https://doi.org/10. 1083/jcb.201409063.

24. Nieto-Vazquez I, Fernández-Veledo S, Krämer DK, Vila-Bedmar R, Garcia-Guerra L, Lorenzo M. Insulin resistance associated to obesity: the link TNF-alpha. Arch Physiol Biochem. 2008;114(3):18394. https://doi.org/10.1080/13813450802181047.

25. Arismendi E, Rivas E, Agustí A, et al. The systemic inflammome of severe obesity before and after bariatric surgery. PLoS One. 2014;9(9). https://doi.org/10.1371/journal.pone.0107859.

26. Könner AC, Brüning JC. Toll-like receptors: linking inflammation to metabolism. Trends Endocrinol Metab. 2011;22(1):1623. https://doi.org/10.1016/j.tem.2010.08.007.

27. Posey KA, Clegg DJ, Printz RL, et al. Hypothalamic proinflammatory lipid accumulation, inflammation, and insulin resistance in rats fed a high-fat diet. Am J Physiol Endocrinol Metab. 2009;296(5):E1003-12. https://doi.org/10.1152/ajpendo.90377. 2008 .

28. Coperchini F, Chiovato L, Croce L, Magri F, Rotondi M. The cytokine storm in COVID-19: An overview of the involvement of the chemokine/chemokine-receptor system. Cytokine Growth Factor Rev. 2020;53:25-32. https://doi.org/10.1016/j.cytogfr. 2020.05.003 Excellent review of the chemokine/chemokine receptor system and how they lead to cytokine storm noted in COVID-19.

29. Yang $\mathrm{X}, \mathrm{Yu} \mathrm{Y,} \mathrm{Xu} \mathrm{J,} \mathrm{et} \mathrm{al.} \mathrm{Clinical} \mathrm{course} \mathrm{and} \mathrm{outcomes} \mathrm{of}$ critically ill patients with SARS-CoV-2 pneumonia in Wuhan, China: a single-centered, retrospective, observational study. Lancet Respir Med. 2020;8(5):475-81. https://doi.org/10.1016/ S2213-2600(20)30079-5.

30. Soy M, Keser G, Atagündüz P, Tabak F, Atagündüz I, Kayhan S. Cytokine storm in COVID-19: pathogenesis and overview of anti-inflammatory agents used in treatment. Clin Rheumatol. Published online May 30, 2020:1-10. doi:https://doi.org/10. 1007/s10067-020-05190-5.

31. Xu Z, Shi L, Wang Y, et al. Pathological findings of COVID19 associated with acute respiratory distress syndrome. Lancet Respir Med. 2020;8(4):420-2. https://doi.org/10.1016/S22132600(20)30076-X.

32. Schnappauf O, Chae JJ, Kastner DL, Aksentijevich I. The pyrin Inflammasome in health and disease. Front Immunol. 2019;10. https://doi.org/10.3389/fimmu.2019.01745.
33. Snijder EJ, van der Meer Y, Zevenhoven-Dobbe J, et al. Ultrastructure and origin of membrane vesicles associated with the severe acute respiratory syndrome coronavirus replication complex. J Virol. 2006;80(12):5927-40. https://doi.org/10.1128/JVI. 02501-05.

34. Bergsbaken T, Fink SL, Cookson BT. Pyroptosis: host cell death and inflammation. Nat Rev Microbiol. 2009;7(2):99-109. https:// doi.org/10.1038/nrmicro2070.

35. Ramos Muniz MG, Palfreeman M, Setzu N, et al. Obesity exacerbates the cytokine storm elicited by Francisella tularensis infection of females and is associated with increased mortality. Biomed Res Int. 2018;2018. https://doi.org/10.1155/2018/3412732.

36. Honce R, Schultz-Cherry S. Impact of obesity on influenza a virus pathogenesis, immune response, and evolution. Front Immunol. 2019;10. https://doi.org/10.3389/fimmu.2019.01071.

37. Paich HA, Sheridan PA, Handy J, et al. Overweight and obese adult humans have a defective cellular immune response to pandemic H1N1 influenza A virus. Obesity (Silver Spring, Md). 2013;21(11):2377. https://doi.org/10.1002/oby.20383.

38. Maier HE, Lopez R, Sanchez N, et al. Obesity increases the duration of influenza a virus shedding in adults. J Infect Dis. 2018;218(9):1378-82. https://doi.org/10.1093/infdis/jiy370.

39. Sheridan PA, Paich HA, Handy J, et al. Obesity is associated with impaired immune response to influenza vaccination in humans. Int J Obes. 2012;36(8):1072-7. https://doi.org/10.1038/ ijo.2011.208

40. Neidich SD, Green WD, Rebeles J, et al. Increased risk of influenza among vaccinated adults who are obese. Int J Obes. 2017;41(9):1324-30. https://doi.org/10.1038/ijo.2017.131.

41. Shang J, Wan Y, Luo C, et al. Cell entry mechanisms of SARSCoV-2. Proc Natl Acad Sci U S A. 2020;117(21):11727-34. https://doi.org/10.1073/pnas.2003138117.

42. Hamming I, Timens W, Bulthuis M, Lely A, Navis G, van Goor $\mathrm{H}$. Tissue distribution of ACE2 protein, the functional receptor for SARS coronavirus. A first step in understanding SARS pathogenesis. J Pathol. 2004;203(2):631-7. https://doi.org/10. 1002/path.1570.

43. Li Y, Zhang Z, Yang L, et al. The MERS-CoV Receptor DPP4 as a candidate binding target of the SARS-CoV-2 Spike. iScience. 2020;23(6):101160. https://doi.org/10.1016/j.isci.2020.101160.

44. Hoffmann M, Kleine-Weber H, Schroeder S, et al. SARS-CoV-2 Cell entry depends on ACE2 and TMPRSS2 and is blocked by a clinically proven protease inhibitor. Cell. 2020;181(2):271-280. e8. https://doi.org/10.1016/j.cell.2020.02.052.

45. Gupte M, Boustany-Kari CM, Bharadwaj K, et al. ACE2 is expressed in mouse adipocytes and regulated by a high-fat diet. Am J Physiol Regul Integr Comp Physiol. 2008;295(3):R781-8. https://doi.org/10.1152/ajpregu.00183.2008.

46. Emilsson V, Gudmundsson EF, Aspelund T, et al. ACE2 levels are altered in comorbidities linked to severe outcome in COVID-19. medRxiv. Published online June 5, 2020:2020.06.04.20122044. https://doi.org/10.1101/2020.06. 04.20122044

47. Higham A, Singh D. Increased ACE2 Expression in the Bronchial Epithelium of COPD Patients who are Overweight. Obesity (Silver Spring). Published online May 19, 2020. https://doi.org/ 10.1002/oby.22907

48. Radzikowska U, Ding M, Tan G, et al. Distribution of ACE2, CD147, CD26 and other SARS-CoV-2 associated molecules in tissues and immune cells in health and in asthma, COPD, obesity, hypertension, and COVID-19 risk factors. Allergy. Published online June 4, 2020. doi:https://doi.org/10.1111/all.14429.

49. Sell H, Blüher M, Klöting N, et al. Adipose dipeptidyl Peptidase-4 and obesity. Diabetes Care. 2013;36(12):4083-90. https:// doi.org/10.2337/dc13-0496. 
50. Shin K, Pandey A, Liu X-Q, Anini Y, Rainey JK. Preferential apelin-13 production by the proprotein convertase PCSK3 is implicated in obesity. FEBS Open Bio. 2013;3:328-33. https:// doi.org/10.1016/j.fob.2013.08.001.

51. Kruglikov IL, Scherer PE. The role of adipocytes and adipocytelike cells in the severity of COVID-19 infections. Obesity (Silver Spring). Published online April 27, 2020. https://doi.org/10. 1002/oby.22856

52. Frat J-P, Gissot V, Ragot S, et al. Impact of obesity in mechanically ventilated patients: a prospective study. Intensive Care Med. 2008;34(11):1991-8. https://doi.org/10.1007/ s00134-008-1245-y.

53. Guérin C, Reignier J, Richard J-C, et al. Prone positioning in severe acute respiratory distress syndrome. $\mathrm{N}$ Engl $\mathrm{J}$ Med. 2013;368(23):2159-68. https://doi.org/10.1056/NEJMoa1214 103.

54. Guérin C, Albert RK, Beitler J, et al. Prone position in ARDS patients: why, when, how and for whom. Intensive Care Med. Published online November 10, 2020:1-12. https://doi.org/10. 1007/s00134-020-06306-w.

55. Elharrar X, Trigui Y, Dols A-M, et al. Use of prone positioning in nonintubated patients with COVID-19 and hypoxemic acute respiratory failure. JAMA. 2020;323(22):2336. https://doi.org/ 10.1001/jama.2020.8255

56. Zarantonello F, Andreatta G, Sella N, Navalesi P. Prone position and lung ventilation and perfusion matching in acute respiratory failure due to COVID-19. Am J Respir Crit Care Med. 2020;202(2):278-9. https://doi.org/10.1164/rccm. 202003-0775IM.

57. Coppo A, Bellani G, Winterton D, et al. Feasibility and physiological effects of prone positioning in non-intubated patients with acute respiratory failure due to COVID-19 (PRONCOVID): a prospective cohort study. Lancet Respir Med. 2020;8(8):765-74. https://doi.org/10.1016/S2213-2600(20) 30268-X.

58. Mechanick JI, Carbone S, Dickerson RN, et al. Clinical Nutrition Research and the COVID-19 Pandemic: A Scoping Review of the ASPEN COVID-19 Task Force on Nutrition Research. Journal of Parenteral and Enteral Nutrition. n/a(n/a). https:// doi.org/10.1002/jpen.2036

Publisher's Note Springer Nature remains neutral with regard to jurisdictional claims in published maps and institutional affiliations. 